(DAPI) stained for cell nuclei was performed. Co-localization of IL-33 and DAPI in CD34 positive cells demonstrates IL-33 staining in the nucleus of glomerular endothelial cell of lupus kidney. Expression of intracellular but not surface ST2 was increased in plasmacytoid dendritic cells/pDC (CD16-CD14-CD85k+CD123 + ) of lupus patients when compared with healthy controls. Incubation of HUVECs with IL-33 and/or IL-12 increased the production of IL-6, but IL-4, IL-5, IL-6, IL-13 and TNF- $\alpha$ was not produced.
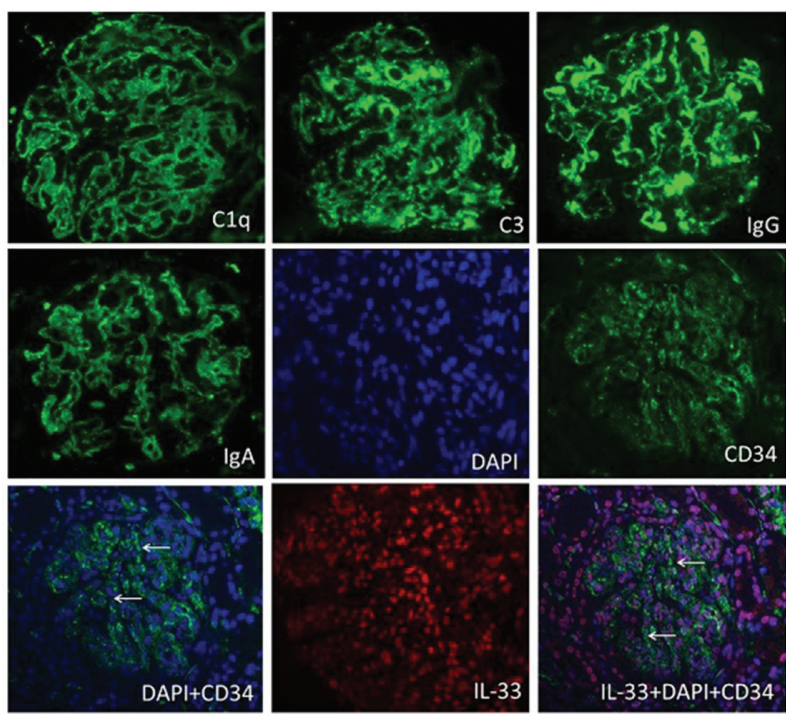

Conclusions: As a result of external stimuli or infection, renal glomerular endothelial cells undergo cellular death and release the "Alarmin", IL-33, to alert the lupus immune system. Released IL-33 interact with their target cells, pDC via their specific receptor ST2 to subsequently induce innate and adaptive responses, activate inflammatory pathways in the pathogenesis of lupus nephritis.

Acknowledgements: This work was supported by National Science Foundation of China (81300585), Science and Technology Planning Project of Guangdong Province (2014A020212322) and Natural Science Foundation of Guangdong Province (2015A030313477).

Disclosure of Interest: None declared

DOI: 10.1136/annrheumdis-2018-eular.6064

\section{SAT0044 LIN28A IS OVEREXPRESSED IN OSTEOARTHRITIS AND IS ESSENTIAL FOR THE STABILITY AND HIGH LEVEL EXPRESSION OF IL-6 AND COX-2 IN HUMAN CHONDROCYTES}

I. Ahmad, M.Y. Ansari, M.N. Khan, T.M. Haqqi. Anatomy and Neurobiology, North East Ohio Medical University, Rootstown, USA

Background: Osteoarthritis (OA), the most common type of joint disease, is characterised by progressive and irreversible degradation of articular cartilage. Dysregulated gene expression has also been linked to disease pathogenesis. Lin28A is an evolutionarily conserved RNA binding protein and known to play a critical role in development, metabolism and tumorigenesis. However, the role of Lin28A in osteoarthritis is not yet explored.

Objectives: To study the role of Lin28A in the regulation of genes associated with OA pathogenesis in human chondrocytes.

Methods: Primary human chondrocytes were isolated from the undamaged portion of the knee OA cartilage by enzymatic digestion and were cultured in DMEM/ F12\% and $10 \%$ FCS. Total RNA from cartilage explants or chondrocytes was prepared using Trizol and was made DNA-free by on-column digestion method. mRNA expression levels of Lin28A, Lin28B, MMP-13, IL-6, COX2 and iNOS were quantified by TaqMan assays. Protein expression was analysed by immuno-blotting using validated antibodies. Nucleofection was used for the siRNA mediated depletion or plasmid mediated overexpression of Lin28A gene was used to study its role in chondrocyte function under pathological conditions. RNA immunoprecipitation (RIP) with anti-Lin28A antibody was performed to identify the interacting mRNA partners. Stability of the mRNAs was determined by Actinomycin-D chase experiments.

Results: Human OA cartilage samples $(n=6)$ analysed were found to express Lin28A but not Lin28B transcripts. Lin28A mRNA expression was significantly high in the damaged $\mathrm{OA}$ cartilage compared to the smooth cartilage from the same patient $(n=3, p<0.05)$. Stimulation with $I L-1 \beta$ induced the high levels of Lin28A mRNA and protein expression in OA chondrocytes in a time dependent as well as dose dependent manner. siRNA mediated depletion of Lin28A expression in $\mathrm{OA}$ chondrocytes inhibited the IL-1 $\beta$-induced expression of MMP-13, IL-6, COX2 and iNOS mRNAs. Importantly, the overexpression of Lin28A induced the expression of MMP-13, IL-6, COX-2 and iNOS mRNA and protein in OA chondrocytes. Immunoblotting analysis showed that the Lin28A depleted OA chondro cytes treated with IL-1 3 also produced significantly low levels of IL-6, MMP-13 and COX-2 protein compared to chondrocytes transfected with scrambled siRNAs $(n=3, p<0.05)$. RIP analyses in IL-1 $\beta$ treated OA chondrocytes with anti-Lin28A antibody revealed that the MMP-13, IL-6 and COX2 mRNAs were pulled down with anti-Lin28A and were highly enriched when compared with the MRNA population pulled down by isotype control antibody. siRNA mediated depletion of Lin28A expression resulted in decreased half-life of IL-6 and COX-2 mRNAs, while the overexpression of Lin28A had the opposite effect in IL-1 $\beta$ stimulated OA chondrocytes. This indicated that Lin28A contributes towards the stability of IL-6 and COX-2 mRNAs.

Conclusions: Our data for the first time demonstrate that Lin28A plays a key role in OA pathogenesis by stabilising the expression of catabolic gene transcripts in human chondrocytes under pathological conditions. These data revealed a previously unidentified role of Lin28A in chondrocytes and identify it as a potential therapeutic target for the treatment of $O A$.

Acknowledgements: Supported by NIH grants RO1 AT-007373, RO1 AR067056 and funds from the Northeast Ohio Medical University to TMH.

Disclosure of Interest: I. Ahmad: None declared, M. Ansari: None declared, M. Khan: None declared, T. Haqqi Grant/research support from: USPHS Grants DOI: 10.1136/annrheumdis-2018-eular.6011

\section{SAT0045 DNA METHYLATION OF SOCS3 AS A POSSIBLE MECHANISM FOR PERSISTENT URATE INDUCED INFLAMMATION}

V. Klück ${ }^{1}$, T. Crisan ${ }^{2}$, M. Cleophas ${ }^{1}$, S. Keating ${ }^{1}$, B. Novakovic ${ }^{3}$, N. Dalbeth ${ }^{4}$, L. Stamp ${ }^{5}$, A. Phipps-Green ${ }^{6}$, M. Black ${ }^{6}$, D. Macartney-Coxson ${ }^{7}$, T. Merriman ${ }^{6}$, L. Joosten'. ' ${ }^{1}$ Experimental Internal Medicine, Radboudumc, Nijmegen, Netherlands; ${ }^{2}$ Department of Medical Genetics, Iuliu Hatieganu University of Medicine and Pharmacy, Cluj-Napoca, Romania; ${ }^{3}$ Department of Molecular Biology, Radboud University, Nijmegen, Netherlands; ${ }^{4}$ Department of Medicine, University of Auckland, Auckland; ${ }^{5}$ Department of Medicine, University of Otago, Christchurch; ${ }^{6}$ Department of Biochemistry, University of Otago, Dunedin; Environmental and Scientific Research, Wellington, New Zealand

Background: Hyperuricemia is a metabolic condition associated with cardiovascular diseases. ${ }^{1}$ However, mechanisms for a causal relation have not been fully elucidated yet. Previously, we showed that monocytes primed with urate show a shift in the balance of cytokine production: increased proinflammatory cytokines and decreased levels of IL-1 receptor antagonist. ${ }^{2}$

Objectives: In this study we investigate if these changes to urate exposure are persistent and whether changes in DNA methylation serve as a molecular substrate for these effects of hyperuricemia.

Methods: DNA methylation was assessed in whole blood of 80 individuals of Maori ancestry with varying serumurate levels. Human PBMCs and monocytes from Dutch healthy volunteers were isolated and pretreated for 24 hour with urate. Cells were either directly stimulated with LPS or LPS/MSU or subjected to increasing resting days before restimulation. Cytokine levels were determined in supernatants by ELISA. SOCS3 mRNA levels were determined by qPCR after 24 hour urate priming. Phosphorylation of STAT3 was assessed after stimulation by flow cytometry with intracellular staining for PSTAT3.

Results: Human PBMCs primed with urate demonstrated increased IL-1 $\beta$ and IL6 responses and decreased IL-1Ra production compared to controls. Although IL$1 \beta$ production was diminished after increasing resting days, persistent effects were observed for the reduction of IL-1Ra and induction of IL-6. To investigate whether these persistent changes were mediated by epigenetic changes, differences in DNA methylation between normouricemic and hyperuricemic individuals were assessed. SOCS3 gene was higher methylated at 3 neighbouring SOCS3 intragenic positions in hyperuricemic individuals. In vitro, SOCS3 mRNA levels were significantly increased in monocytes after 24 hour urate treatment. Moreover, urate dose-dependently suppressed the phosphorylation of STAT3 after stimulation.

Conclusions: In this study we demonstrated that urate has persistent proinflammatory effects on human monocytes. Higher SOCS3 DNA methylation is observed in hyperuricemic individuals. In vitro, urate priming leads to increased levels of SOCS3 mRNA and consequently suppression of STAT3 phosphorylation. Interestingly, STAT3 inhibition has been reported to mediate IL-1Ra downregulation. ${ }^{3}$ Therefore, we hypothesize urate induced inflammation is at least partly mediated by changes in methylation of the SOCS3 gene. However, further validation of this pathway is needed to elucidate possible targets for therapy. 